This document is published in:

Wolpers, M. et al. (eds.) (2010) Sustaining TEL: From Innovation to Learning and Practice: 5th European Conference on Technology Enhanced Learning, EC-TEL 2010, Barcelona, Spain, September 28 - October 1, 2010. Proceedings. (Lecture Notes in Computer Science, 6383). Springer, 139-150.

DOI: http://dx.doi.org/ 10.1007/978-3-642-16020-2_100-2_10

(C) 2010 Springer-Verlag Berlin Heidelberg 


\title{
Management of Assessment Resources in a Federated Repository of Educational Resources
}

\author{
Israel Gutiérrez Rojas, Derick Leony, Andrés Franco, Raquel M. Crespo, \\ Abelardo Pardo, and Carlos Delgado Kloos \\ Universidad Carlos III de Madrid, Telematic Engineering Department, \\ Av. de la Universidad 30, 28911 Leganés (Madrid) - Spain \\ \{igutierrez, afranco\}@inv.it.uc3m.es, \\ \{dleony, rcrespo, abel, cdk\}@it.uc3m.es
}

\begin{abstract}
This article tries to shed some light over the management of assess-ment resources in a repository of educational resources from an outcome-based perspective. The approximation to this problem is based on the ICOPER Refer-ence Model, as a model to capture e-learning data, services and processes, ad-dressing an interoperability approach. To demonstrate this proposal, a prototype has been implemented. This article also describes the design and development of this prototype that accesses a repository of educational resources (the Open ICOPER Content Space - OICS), the main features of the prototype, the development environment and the evaluation that is being performed.
\end{abstract}

Keywords: assessment resources, .LRN, federated repository, IMS QTI, OICS.

\section{Introduction}

Sharing assessment resources among teachers and course developers is a feature that has a great potential. The exposition in this article tries to shed some light over this feature and provides a solution by means of an application prototype. The learning management systems (LMSs) are the entry point for the mentioned stakeholders to the educational resources used by an institution. A possible solution for sharing assessment resources among learning management systems (LMSs) would be to use an information repository, such as the Open ICOPER [1] Content Space (henceforth referred to as OICS). By doing so, assessment content and information could be centralized and the material would be accessible from any other platform. All contents could be downloaded from a single centralized site, thus simplifying the integration of different LMS and interoperability.

There have been some initiatives for creating Open Educational Resources (OER) repositories, like the JISC OER program [2], which purpose is "make a significant amount of existing learning resources freely available online, licensed in such away to enable them to be used and repurposed worldwide." Some of these repositories contain assessment resources in several formats (e.g., IMS QTI [3]), like Fetlar [4] that collect maths assessment using IMS QTI and Bioscience UKOER project, whose resources can be accessed through JorumOpen [5].

In order to realize the exchange of assessment resources, a common assessment specification must be used. Although IMS QTI 1.2.1 presents deficiencies and limitations, as 
discussed in [6], it is nevertheless considered a de facto standard by the industry. It is also the most popular version nowadays and the one currently endorsed by the IMS Global Learning Consortium. Thus, this is the specification selected in order to widen the scope of the repository, accomplish universal access and allow it to provide services for both authoring tools as well as LMSs.

One important ICOPER task is to corroborate that the assessment model proposed in [7] fits properly in the ICOPER Reference Model (IRM). It is also needed to validate that the proposed model can support any kind of assessment scenario that a higher education institution may present. The process to perform this validation consisted of the development of a prototype of an assessment application that connects to the OICS. This prototype would help to demonstrate that the proposed IEEE LOM [8] profile, presented in the third section, provides a proper set of metadata information to exchange and work with learner assessments.

The ICOPER Reference Model (IRM) is a model based on the state-of-the-art in standards and specifications in the field that support learning outcome-driven content management in Technology-Enhanced Learning (TEL). The main objectives of this conceptual model are [9]:

- To describe the domain of outcome-based, technology-enhanced learning.

- To illustrate the structure of the reference model to support stakeholders to develop, use and improve (information and communication) systems for outcome-based learning.

- To initiate a discourse process on the reference model.

- To incorporate best practices to a common body of knowledge around this model.

The model consists of the following levels:

- Processes: key processes for the development, use, and improvement of outcome-based learning.

- Services: a classification and a description format as well as a collection of (technical) services that can be incorporated when developing outcomebased learning systems.

- Data: data models for data and information exchange between teaching and learning systems to improve interoperability.

This article is structured as follows: after the introduction of the problem and the ICOPER reference model, in section 2 the learner assessment is presented in the context of the IRM; after that, section 3 provides a perspective of the learner assessment and related concepts from the point of view of interoperability and proposes a metadata model for complementing the assessment resources; section 4 deals with the design and implementation of a prototype that permits teachers and course developers to manage and share assessment resources; finally, section 5 presents the conclusions of this work and the future works.

\section{Learner Assessment in the ICOPER Reference Model}

According to the ICOPER reference model presented in the previous section, the role of the learner assessment in the different levels is the following. 
Processes. Two key processes have been identified related to the assessment process. The first one is the "Assessment planning" carried out by the learning supporter, i.e., a teacher or instructor. It consists of searching assessment resources that cover the intended learning outcomes of the learner. If the learning supporter finds a suitable resource (i.e., that addresses intended outcomes) from existing repository, it will be reused; otherwise, the learning supporter will create a new assessment resource. After that, the resources could be annotated, e.g., with student performance, and published if the learning supporter decides to do it. This process is represented in Fig. 1.

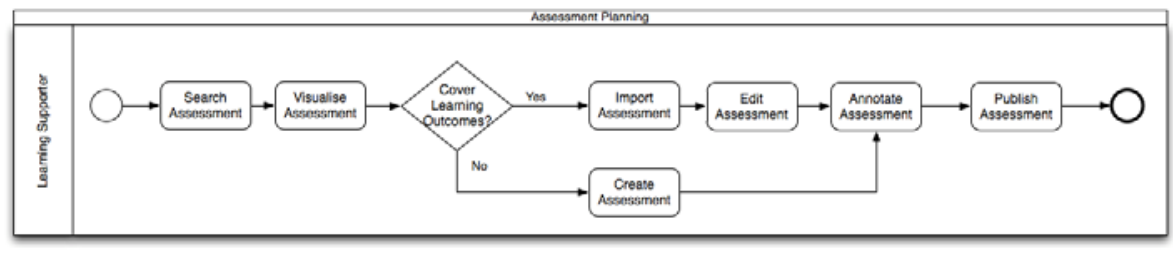

Fig. 1. Assessment planning process

The second process is the "Learner Assessment Activities" and describes the activities carried out by the learning supporter and the learner during the assessment process runtime. The delivery process is driven by the learning supporter, which allows the learner to visualize and answer the assessment, and then submit the response. Then is the turn of the learning supporter to appraise the learner response what generates some assessment results that could be visualized by the learner. This process is represented in Fig. 2.

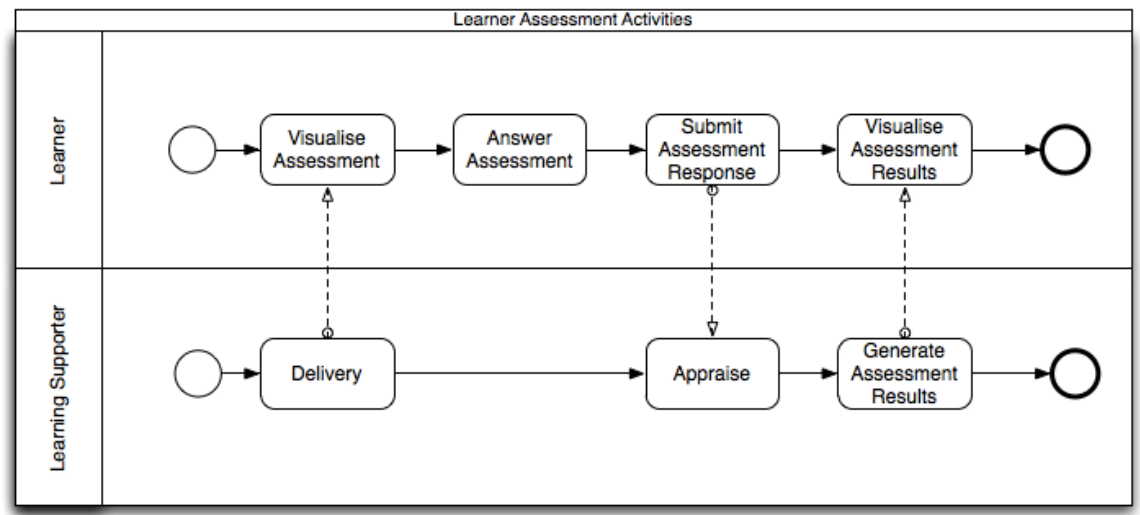

Fig. 2. Learner Assessment Activities process

Services. Three generic services have been identified for assessment purpose. These are a) the search and retrieval of resources, i.e., a service that allows search of assessment resources by some filter parameters; b) the publication of resources, i.e., a 
service that allows to publish resource information in a common shared virtual space; and c) a repository service, i.e., a service that allows storage of resources themselves. These generic services could be used as reference in concrete assessment use cases.

Data. At this level, there are two different parts to mention. The first one is an IMS QTI profile that is being developed in the ICOPER project as a best practice result. This profile is being developed taking into account the different implementations of the specification in European institutions. The second one is based on the relation of the assessment process to other e-learning aspects, like learning outcomes and units of learning. This result is presented in the next section as an IEEE LOM profile.

\section{Learner Assessment Interoperability}

The assessment interoperability proposal made in this section is twofold: on one hand, a connection to other e-learning concepts has to be achieved; on the other, a set of recommendations to simplify the IMS QTI usage has to be made due to the problems found in [6], like flexibility handicaps, version instability and incomplete support in assessment tools.

\subsection{Learner Assessment Related Concepts}

The IMS QTI data model does not include elements for representing or linking to concepts related to other learning aspects of the ICOPER Reference Model (IRM). For example, the unit of learning, the learning outcome or the assessment record are concepts that are related to assessment but not supported by IMS QTI data model. On the other hand, IMS QTI includes information about assessment resources (data level), but it does not provide information of learner assessment, like context (i.e., the set of educational elements that is external to and adds meaning to an achieved learning outcome or Learning Opportunity [9]). Learner Assessment was defined as the process of testing the learning outcomes (knowledge, skills and/or competences) attained by an individual learner and providing the corresponding information reporting about the student achievements and/or potential indications for improving them. As explained in [10], in Higher Education contexts it comprises identifying, collecting and preparing data to evaluate the achievement of program outcomes and program educational objectives.

The integration of assessment concepts and other e-learning process building blocks translates into a series of benefits. For example, the relation of learner assessment to learning outcomes results in the possibility of searching learner assessments in a repository based on the intended learning outcomes. On the other hand, the relation to units of learning stored in a repository results in getting assessment material attached to a specific unit of learning. Finally, the relation to assessment records (through assessment results) is necessary to access grades and feedback achieved by a learner attached to a particular assessment record, e.g., a certificate, and to update the learner profile or PALO (Personal Achieved Learning Outcomes) [11].

In Fig. 3, the Learner Assessment (LA) concept has been defined as an entity that contains all the data related to the assessment process. It includes the assessment resources and implements one or more assessment methods. Besides, the Unit of Learning 
(UoL) is related to the Learner Assessment used in the UoL. Finally, the assessment results (using IMS QTI format) will be normalized into assessment records; this part is still work in progress in the context of ICOPER. The relation between learning methods (that include Teaching Methods and Assessment Methods, that are a special type of them) and the intended learning outcome definitions (LOD) is not going to be developed in the OICS prototype. The main reason is that the real linkage to the learning outcomes is already done from the unit of learning/learner assessment (contextualized versions of the learning methods). The only types of learning outcomes missing are the generic skills (e.g. presentation skills), which are acquired in several UoL/LA, but they are not the main objective of the UoL. An in depth research will be performed to determinate how to deal with this type of learning outcomes.

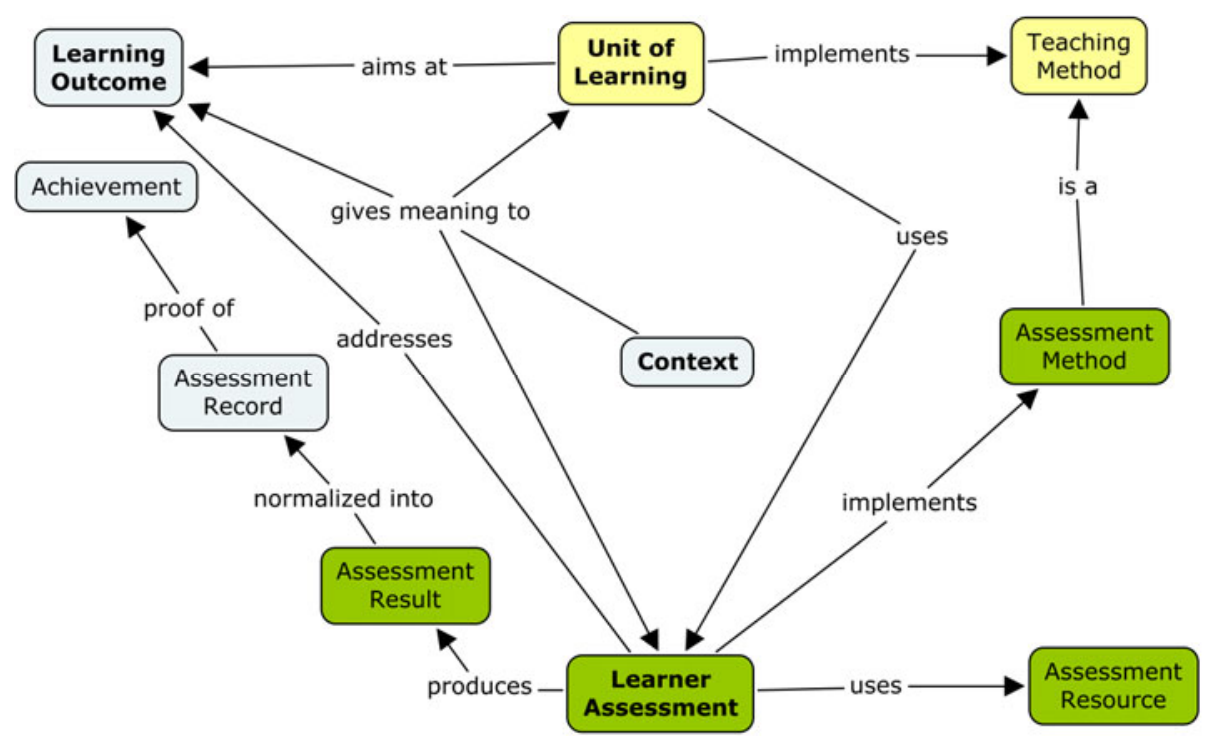

Fig. 3. Learner assessment and related concepts

Another concept not covered by the previous analysis is the assessment method. The assessment method describes the assessment methodology applied, completely specifying all the features that characterize the different dimensions of the assessment process. As explained in [12], there are several dimensions to define assessment methods like:

- Objective: summative or formative assessment.

- Collaboration: individual or collaborative assessment.

- Assessor: instructor-based, computer-based, peer or self-assessment.

- Channel: writing, oral, online, simulation assessment.

- Activity type: collaboration, questions and answers (MCQ, FIB, short answer), etc. 
These list of assessment method dimensions is not complete, that is, there could be some other dimensions that make sense in a concrete assessment scenario. But the dimensions concept helps to define new assessment methods.

\subsection{Interoperable Assessment Resources}

In this subsection a LOM profile is presented that allows the connection of the assessment resources (e.g., using IMS QTI) to other e-learning aspects, like learning outcomes and learning designs. Implementing this profile guarantees high-level assessment interoperability, i.e., interoperable assessment services and processes.

Given these ideas, the IMS QTI data model should be extended in order to provide it with these connections, as explained in [6]. The new data model should include information of assessment resources, but also define the context and the process of the learner assessment. Two possibilities arose in order to perform this extension:

- Extending IMS QTI (what would be called X-QTI) to include new metadata using IMS QTI current concepts, e.g., the new data will be included in the same QTI file. Besides, a QTI schema should be defined to present this new utilization of elements.

- Using a complementary standard for metadata representation, such as IEEE LOM or Dublin Core, that covers the concepts that IMS QTI does not cover, e.g., relation between learner assessment and a unit of learning. The main problem of choosing this option is that some metadata attributes could be duplicated in the selected standard and IMS QTI.

The second option has been chosen because the extension of IMS QTI should be transparent for the system, i.e., current IMS QTI resources could be reused. For example, a system importing assessment data formatted in X-QTI and not supporting the defined schema should ignore extended data and will import just the assessment content in a proper way. But the current implementations in LMSs could not perform this behavior.

Due to that, it was decided to define the additional metadata using a metadata schema, in concrete, an IEEE LOM profile. The selection of IEEE LOM to represent assessment metadata was based on these reasons:

- It is a mature and widely adopted standard for learning objects metadata and allows connection to other e-learning domain concepts using the Relation attributes (for existing learning objects such as UoLs) and extensions (for nonLOM ones, like LODs).

- IEEE LOM relation to IMS QTI: there is a LOM profile defined in IMS QTI 2.X which the learner assessment profile could be based on.

- Homogeneity with other learning object metadata definitions, i.e., UoLs and TM in D3.1 [13]. In this document, the advantages of using IEEE LOM instead of Dublin Core (DC) or Dublin Core Educational for describing UoL and TM are exposed. Following the same reasoning, Dublin Core proposes a too general set of metadata elements; besides, Dublin Core Educational (a DC application profile for education) requires also some extensions to represent the assessment concepts. 
- The IEEE LOM profile is already implemented in the OICS thus facilitating compliance and interoperability with other learning aspects.

The LOM profile proposed for ICOPER learner assessment metadata is defined in [13]. The main concepts of learner assessment are mapped into IEEE LOM profile as follows:

- Learner Assessment is defined by all LOM attributes and categories, including annotations (LOM Learning Resource Type: "Learner Assessment"). The learning resource type "Learner Assessment" is identified as the "Assessment Item" of Resource Discovery Network/Learning and Teaching Support Network (RDN/LTSN) resource type vocabulary [14]. This vocabulary is recommended in the IMS QTI profile.

- Learning resources are presented as IMS QTI files.

- Assessment Methods are related to Learner Assessment using IEEE LOM Relation.

- Units of Learning are related to Learner Assessment through IEEE LOM Relation.

Annotations could be done using the annotation part of LOM. In IMS QTI 2.X there are also mechanisms to define statistics of usage, but they will not be used to make it simpler.

Related to assessment methods, the implementations in the defined LOM profile are as follows:

- An instance of learner assessment can use one or more assessment methods. For this reason, the LOM Relation element will be used to link both concepts.

- An assessment method (AM) is a specialization of a teaching method (TM). Thus, an AM can be described using the same metadata. Besides, the IEEE LOM Educational Learning Resource Type (LRType) for a method instances could be AM, TM or both. Given a learning method, e.g., peer review, it could be considered as a TM and as an AM at the same time. That is the reason to allow multiple learning resource types.

In order to model this concept, IEEE LOM standard was used to describe its metadata elements in the OICS. As the assessment method concept inherits from the teaching method concept, the LOM profile used is the same for TM defined in [13].

\section{Prototype Design and Implementation}

The design phase of the .LRN prototype is based in a set of use cases. The use cases cover the services of the ICOPER Reference Model and the assessment planning process.

As the developed prototype is intended for assessment resources management, a set of assessment use cases have been implemented in the .LRN module.

\subsection{Scenarios to Be Supported by Prototype Application}

The design of the prototype for the assessment application was based on a set of assessment scenarios. This set contains three scenarios that involve the use of learner 
assessments during the design and enactment phases of a course. The scenarios taken into account for this set are the following:

1. Reuse of assessment resources available in the OICS.

2. Annotation of assessment resources by a learning supporter from a LMS.

3. Publication of assessment resources to the OICS from a LMS or authoring tool.

In order to provide a broader view of the process to validate the application prototype, an explanation of each one of these scenarios and their implications is presented as follows.

Scenario: Reuse of assessment resources during design phase. The first scenario is the reuse of an assessment resource during the Unit of Learning (UoL) design. This scenario describes a learning supporter that is planning the learner assessment that takes part of a UoL. He/she will search for assessment resources in the OICS according to a series of parameters like:

- The intended learning outcomes (knowledge, skill, competence) of the assessment

- The assessment method (e.g., type - summative, formative, diagnostic; or by assessor: instructor, peer, self or automatic).

The learning supporter could also search resources by language, author/contributor, date, name, description, format (e.g. PDF, MS Word Document), and rights (e.g.. Creative Commons - Share Alike).

The workflow of this scenario is described as follows:

1. Search assessment resources. This search can be based on keywords, learning outcomes or assessment methods.

2. Retrieval and review of results. The result view includes this information about the learner assessment: title, language, date, description, format and rights.

3. Selection of assessment resource.

4. Import of assessment resource into a course of the LMS.

Scenario: Annotation of assessment resources. The second scenario is the annotation of assessment resources by any teacher that has used the assessment resource in a real context. This scenario describes a learning supporter that wants to annotate assessment resources with information of students' average performance on the assessment. For example, the learner supporter wants to annotate a multiple choice question (MCQ) with the percentage of students who had selected each of the options. This information can help to detect ambiguous questions and also concepts particularly difficult for the students. Thus, this information can be applied by the learning supporter him/herself or other colleagues during the planning and design phase of the next course. These annotations contain date and time information (generated automatically), and possibly some data about the course in which the information was gathered. Annotation could be used also for peer reviewing of assessment resources. 
Scenario: Publication of learner assessments to the OICS. The last scenario is the publication of assessment resources to the OICS. In this case, a learning supporter wants to publish assessment resources to the OICS. To do so, the required metadata should be aggregated to the assessment resource. Therefore, the only needed step in the workflow of this scenario consists of describing assessment resources with the corresponding metadata. For example, including information about intended learning outcomes, assessment method (e.g., type - summative, formative, diagnostic; the assessor: instructor, peer, self or automatic), language, author/contributor, date, name, description, format (e.g. PDF, MS Word document), and rights (e.g. Creative Commons - Share Alike).

One example of this scenario is the following: the assessment author has already created some assessment resources in the LMS (e.g. .LRN). This LMS supports the IMS QTI assessment specification to create and store the resources. When the author decides to share resources he has to annotate them with metadata, e.g., defining intended learning outcomes, defining who the author of the resource is and indicating that the assessor for this assessment should be an instructor, because this cannot be done automatically.

\subsection{Use Cases Abstracted from Scenarios}

The scenarios presented in the previous sub-section allow the abstraction of a set of use cases that can be aggregated into two:

- $\quad$ Search of learner assessments

- Publication of learner assessments

Use case: Search of learner assessments. The application prototype, which has been developed as a module of the .LRN LMS, supports the first use case: search of learner assessments. It also completely fulfils the first scenario described in this section, thus validating the general conceptual model for the exchange of learner assessments in a real scenario.

The implementation of this use case has been possible with a high level of simplicity due to the provision of an intermediate layer between the systems and the OICS. This intermediate layer has been defined as the Middle Layer API and is being documented in the deliverable D1.2 [15]. Basically, it provides a series of services needed for learning object management: search, publication, storage, etc.

Use case: Publication of learner assessments. Regarding the publication of learner assessments, the architecture and functionality of the OICS permits the publication of assessment resources enriched with IEEE LOM metadata. The mentioned metadata fields allow users to include information that is not present in the IMS QTI resources, like intended learning outcomes, teaching methods used and annotations. The union of assessment resources and metadata is called learner assessment (LA).

Regarding to the publication possibilities that the OICS permits, the institutions could share their own repositories by means of the OAI-PMH protocol [16]. But the option chosen for this development is to upload the assessment resources directly to the OICS by means of some middle layer API methods created for this purpose, because the University Carlos III of Madrid does not have already a repository of assessment resources. 


\subsection{Creation of Mockups}

In order to define the concrete functionalities that the prototype was going to implement, a set of mockups was created before the actual implementation. These mockups are screenshots of the main important pages of the application and show the main functionalities and the user interface. An example of a mockup for this prototype is shown in Fig. 4. These application mock-ups were not evaluated just after their production, but when the functional prototype was evaluated.

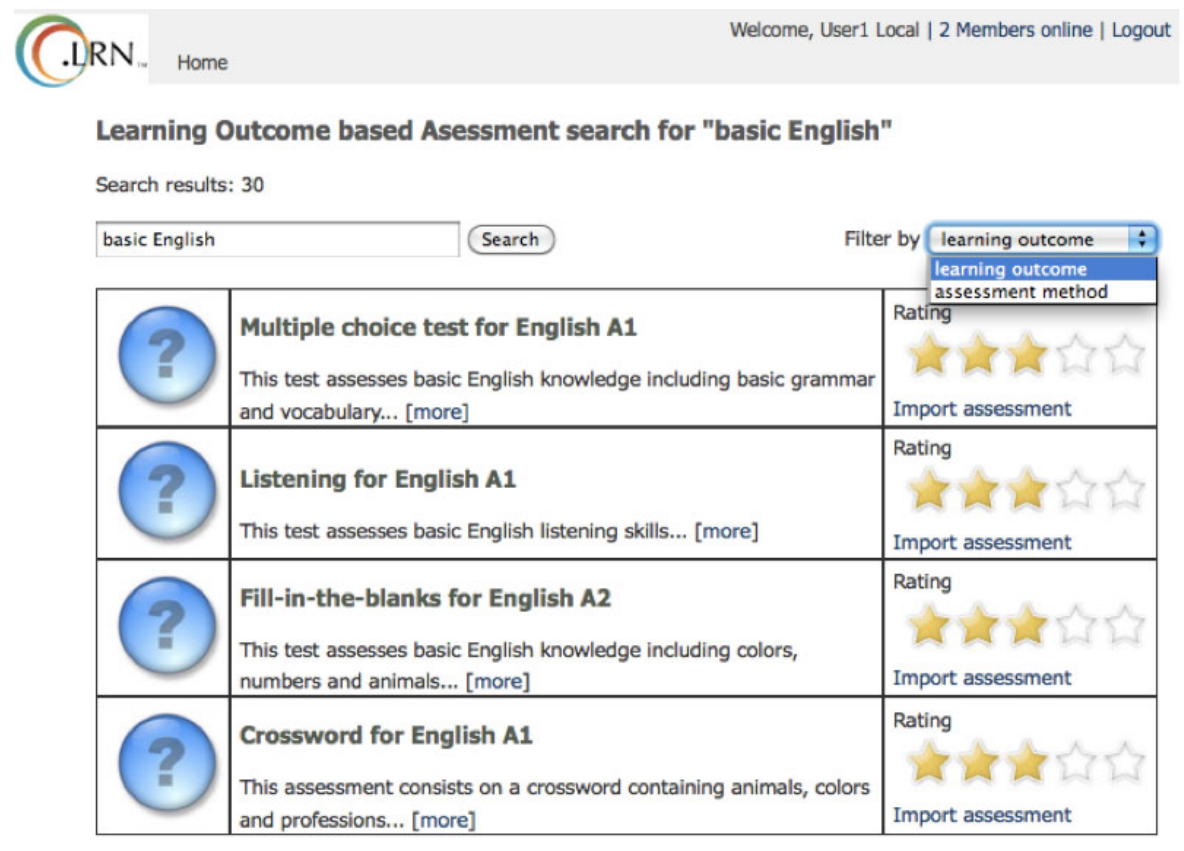

Fig. 4. Mockup of the search page of the .LRN prototype

\subsection{Prototype Environment}

The prototype has been implemented as a .LRN package. As stated in the .LRN website [17], .LRN is the world's most widely adopted enterprise-class open source software for supporting e-learning and digital communities. Originally developed at MIT, .LRN is used worldwide by over half a million users in higher education, government, non-profit, and K-12.

The selection of .LRN as the learning management system for the OICS prototype development has been quite clear, because this platform provides us with the required features for this development. For instance, it already has a package for authoring and managing assessment resources using IMS QTI 1.2.1, so the implemented prototype 
could make use of these capabilities. Another reason to choose this framework was the expertise and satisfactory results obtained from previous experiences with the platform in other research projects.

\subsection{Prototype Evaluation}

The prototype evaluation, according to the methodology defined in the ICOPER project, targets three types of audience (stakeholders):

- Engineering evaluation targeted to implementers, tool developers and technology providers.

- End-user evaluation targeted to instructors, learners, curriculum developers, administration or management.

- Epistemological evaluation targeted to researchers and standardization bodies.

The first one consists of a survey about the prototype implementation and the middle layer API. It will provide the perspective of these stakeholders from a technical point of view.

In the presented prototype, the end-user evaluation targets instructors and curriculum developers. It also consists of a survey that provides us the opinion about usability and utility of this targeted audience. This evaluation will be carried out in several training events, like ECTEL 2010.

\section{Conclusions and Future Work}

This article has presented the design and implementation of an application prototype that permits teachers and course developers manage and share assessment resources in an easy way. The development of the prototype also helped to prove that an application could connect and interact easily with a federated repository of educational resources, the Open ICOPER Content Space, through the use of the Middle Layer API.

The implemented prototype also satisfies the requirements established by the presented scenarios and use cases, so it is supposed to fulfill stakeholders' requirements in the assessment process.

On the basis of the experience gained from the development of this prototype, a series of recommendations for assessment interoperability have been proposed. These recommendations are related to the idea of extending the IMS QTI specification in order to link it to other e-learning fields. This integration brings some benefits, which emerge in the context of e-learning material repositories with several types of content, i.e., learner assessments, units of learning, learning outcomes, etc.

As future work, this prototype will be evaluated by relevant stakeholders like assessment authors (en users, like teachers and course developers) and implementers of learning tools, in order to obtain feedback about the proposed design and workflow.

A proposal of an IMS QTI profile will be done that will contain just a minimum set of elements that guarantee interoperability, based on the study of usage of the specification and stakeholders' needs in [12]. For example, features such as adaptive questions and templates are not implemented in the studied LMSs (Moodle and .LRN) [7], so the recommendation will be not to use them. 
Acknowledgments. This work was partially funded by the Best Practice Network ICOPER (Grant No. ECP-2007-EDU-417007), the Learn3 project, "Plan Nacional de I+D+I" TIN2008-05163/TSI, and the eMadrid network, S2009/TIC-1650, "Investigación y Desarrollo de tecnologías para el e-learning en la Comunidad de Madrid".

\section{References}

1. ICOPER project, http: / /www. icoper.org

2. JISC UK OER program, http: / /www. jisc.ac.uk/oer

3. IMS Global Consortium: IMS Question \& Test Interoperability Specification, http: / / www.ims.org/question/

4. Fetlar project, http: //www. fetlar.bham.ac.uk/

5. JorumOpen, http: // open.jorum.ac.uk/xmlui

6. Gutiérrez Rojas, I., Agea, A., Crespo, R.M., Pardo, A., Leony, D., Delgado Kloos, C.: Assessment interoperability using QTI. In: ICL 2009 Conference, Villach, Austria, September 23-25 (2009)

7. Agea, A., Crespo, R.M., Delgado Kloos, C., Gutiérrez Rojas, I., Leony, D., Pardo, A.: Production flow description and prototype for the two platforms under study (Moodle and.LRN) including the required steps to exchange the material in both platforms. ICOPER deliverable D6.2 (February 2010),

http: / / icoper.org/results/deliverables

8. IEEE LOM, http: / / Itsc. ieee.org/wg12/

9. Kozlov, D., Pulkkinen, M., Pawlowski, J.: ICOPER Reference Model IRM. Conceptual Model. ICOPER deliverable D7.1 (October 2009)

10. ABET: Accreditation Policy and Procedure Manual (2008-2009)

11. Najjar, J., et al.: ISURE: Model for describing learning needs and learning opportunities taking context ontology modelling into account. ICOPER deliverable D2.1 (January 2010) http: / / icoper.org/results / deliverables

12. Agea, A., Crespo, R.M., Delgado Kloos, C., Gutiérrez Rojas, I., Leony, D., Pardo, A.: Analysis of existing specifications and standards for assessment and evaluation and their usage in Europe. ICOPER deliverable D6.1 (October 2009), http: / / icoper.org/results/deliverables

13. Derntl, M., Neumann, S., Oberhuemer, P.: Report on the Standardized Description of Instructional Models. ICOPER deliverable D3.1 (September 2009),

http: / / icoper.org/results / deliverables

14. RDN/LTSN resource type vocabulary, http: / / www. intute.ac.uk/publications/ran-ltsn/types /

15. Totschnig, M., et al.: Open ICOPER Content Space Implementations of 2nd Generation of Open ICOPER Content Space including Integration Mini Case Studies. ICOPER deliverable D1.2 (Work in progress, September 2010)

16. The Open Archives Initiative Protocol for Metadata Harvesting, http: / /www . openarchives . org/OAI / openarchivesprotocol.html

17. LRN, http://dotlrn.org 